\title{
Hedging bounded claims with bounded outcomes
}

\author{
Freddy Delbaen ${ }^{1}$ \\ ETH Zürich, Department of Mathematics, CH-8092 Zurich, Switzerland
}

\begin{abstract}
We consider a financial market with two or more separate components each driven by a Brownian Motion. We look at the problem to hedge a bounded contingent claim in such a way that all the components remain bounded. The problem can also be rephrased as a problem in risk measures.
\end{abstract}

Key words: Hedging, infimal convolution, coherent utility functions, Fatou property, Brownian Motion

Mathematics Subject Classification (2000): 60G44

\footnotetext{
${ }^{1}$ The author thanks Credit Suisse for the support. Only the author is responsible for the contents of this paper.
} 


\section{Introduction and Notation}

We use standard notation from Brownian Motion theory. For any unexplained notion we refer to [4]. We suppose that $B$ is a standard Brownian motion taking values in space $\mathbb{R}^{d}$. The process $B$ is defined on a filtered probability space $\left(\Omega,\left(\mathcal{F}_{t}\right)_{t}, \mathbb{P}\right)$. We suppose that the filtration $\mathcal{F}$ is generated by the Brownian motion augmented with the null sets. The $\sigma$-algebra $\mathcal{F}_{\infty}$ whenever needed, is the $\sigma$-algebra generated by the process $B$ together with all the null sets. Stochastic integration is with respect to the Itô integral. When we use processes that are predictable we mean predictable with respect to the filtration $\mathcal{F}$. Of course in case of a Brownian motion, there is no difference between predictable processes and optional processes. The Brownian motions $B^{j}$ are just the coordinates of the process $B$. We remark that the processes $B^{j}$ are independent. With these processes we will construct correlated Brownian motions $\eta^{1}, \ldots, \eta^{d}$. These processes generate subspaces of $L^{\infty}$ defined as the outcomes of stochastic integrals that remain bounded, more precisely

$$
W_{j}=\left\{\left(H \cdot \eta^{j}\right)_{\infty} \mid H \text { predictable and } H \cdot \eta^{j} \text { bounded }\right\} .
$$

The spaces are weak* closed subspaces of $L^{\infty}$. We will also use the sets

$$
\mathcal{A}_{j}=W_{j}+L_{+}^{\infty},
$$

where $L_{+}^{\infty}$ denotes the cone of nonnegative random variables. It is well known that also the cones $\mathcal{A}_{j}$ are weak* closed, see [3], [2]. The main result of the paper states that under a condition for the correlation matrix of $\eta$, more precisely assumption Delta below, the norm-closure of $\sum_{j} \mathcal{A}_{j}$ is equal to the set $\{f \mid$ $\mathbb{E}[f] \geq 0\}$. As an example will show, the space $\sum_{j} W_{j}$ is not norm closed and its norm closure is not equal to $\{f \mid \mathbb{E}[f]=0\}$. This means that there are random variables $a \in L^{\infty}$ such that $\mathbb{E}[a]=0$ but these random variables cannot be approximated in $L^{\infty}$ norm by sums of bounded stochastic integrals with respect to $\eta$. If however we allow sums of bounded stochastic integrals and nonnegative random variables, then such elements $a$ can be approximated in $L^{\infty}$ norm. The example will make use of the theory of $B M O$ martingales, see [4] for details on $B M O$.

We will suppose that the individual processes $\eta^{j}$ are normalised. So we make the assumption that $d\left\langle\eta^{j}, \eta^{j}\right\rangle=d t$. The correlation matrix $\Sigma$ of $\eta$ is defined through the equation $\left(\Sigma_{t}\right)_{i, k} d t=d\left\langle\eta^{i}, \eta^{k}\right\rangle_{t}$. The Kunita-Watanabe inequalities (see [4]) allow to write the brackets this way. Of course we suppose that $d t \times d \mathbb{P}$ almost everywhere, the matrix $\Sigma$ is symmetric and nonnegative definite. Furthermore $\Sigma$ is predictable. The normalisation simply means that the diagonal terms of $\Sigma$ are equal to 1 . The following assumption is critical.

Assumption Delta: We suppose that there is a real number $\delta>0$ so that the eigenvalues of $\Sigma$ are bounded below by $\delta$. This means that a.e.

$$
\left(x^{1}, \ldots, x^{d}\right) \Sigma\left(x^{1}, \ldots, x^{d}\right)^{\prime} \geq \delta \sum_{j}\left(x^{j}\right)^{2} .
$$


In other words, we have that $\Sigma$ is uniformly elliptic. Furthermore we assume that $d \eta=\Sigma^{1 / 2} d B$ where $\Sigma^{1 / 2}$ is a positive definite square root of $\Sigma$ and where $B$ is the given standard Brownian motion. The reader can see that the latter assumption is not essential.

The approximation result can be seen as an hedging property. To explain this and to see the difference between the usual hedging property, let us observe (see the next section for the details) that every random variable $a \in L^{\infty}$ can be written as $a=\mathbb{E}[a]+(H \cdot \eta)_{\infty}$ where $H$ is a predictable $\mathbb{R}^{d}$ dimensional process and the stochastic integral $H \cdot \eta$ has to be seen as a vector stochastic integral and $H \cdot \eta$ is a bounded process. This means that it is a more general construction then just taking the sum of the individual stochastic integrals $H^{j} \cdot \eta^{j}$. The latter integrals are not necessarily bounded but the sum $\sum_{j} H^{j} \cdot \eta^{j}$ is. The approximation result can be rewritten as

$$
\mathbb{E}[a]=\inf \left\{\alpha \in \mathbb{R} \mid a \leq \alpha+\sum_{j} f_{j} \text { where } f_{j} \in W_{j}\right\} .
$$

In other words even if the random variable $a$ cannot be approximated by a sum of the form $\alpha+\sum_{j}\left(H^{j} \cdot \eta^{j}\right)_{\infty}$ where each $H^{j} \cdot \eta^{j}$ is bounded, it can be approximated by such sums where nonnegative functions have been added/subtracted.

In finance the admissible integrands play a special role. We will not give definitions but just recall that for our purpose a predictable process $H$ taking values in $\mathbb{R}^{d}$ (or in $\mathbb{R}$ depending on the context) is said to be admissible (admissible for $\eta^{j}$ ) if the process $H \cdot \eta$ (or $H \cdot \eta^{j}$ ) is uniformly bounded from below. Replacing the requirement that each process $H^{j} \cdot \eta^{j}$ is uniformly bounded from below does not change the situation. Indeed if each $H^{j}$ is admissible with respect to $\eta^{j}$ and if the sum $\sum_{j} H^{j} \cdot \eta^{j}$ is bounded (from above and from below), say by 1, then each $H^{j} \cdot \eta^{j}$ is bounded. To see this let us suppose that each $H^{j} \cdot \eta^{j}$ is bounded from below by a constant $-m$. Then each $H^{j} \cdot \eta^{j}$ is bounded from above by $(d-1) m+1$.

\section{Technical Preliminaries}

In this section we recall a technical result from stochastic analysis. This result will be applied in the next section where the proof of the main result will be given.

Lemma 1 Under assumption Delta we have that $\eta$ has the representation property. More precisely each local martingale $X$ is of the form $X=H \cdot \eta$, where

for each $t, \int_{0}^{t}\left|H_{u}\right|^{2} d u<\infty$. Conversely each such process $H$ defines a local martingale.

Proof The proof follows easily from linear algebra. The norm of the matrix $\Sigma$ is uniformly bounded since it is positive definite and has diagonal elements equal to 1. Furthermore assumption Delta implies that also $\Sigma^{-1 / 2}$ is uniformly bounded. Since $d \eta=\Sigma^{1 / 2} d B$, it follows that $d B=\Sigma^{-1 / 2} d \eta$. Since $B$ has the 
representation property we get that $Z$ is $B$ integrable if and only if $Z^{\prime} \Sigma^{-1 / 2}$ is $\eta$ integrable if and only if for each $t, \int_{0}^{t}|Z|^{2} d u<\infty$ almost surely.

Lemma 2 Suppose assumption Delta holds for $\eta$. If $H^{n}$ is a sequence of $\eta$ integrable $\mathbb{R}^{d}$ valued predictable processes, if $\left(H^{n} \cdot \eta\right)^{*}=\sup _{t}\left|\left(H^{n} \cdot \eta\right)_{t}\right|$ tends to zero in probability, then $\int_{0}^{\infty}\left|H_{u}^{n}\right|^{2}$ du tends to zero in probability. Also each $\left(H^{n, j} \cdot \eta^{j}\right)_{\infty}$ tends to zero in probability. Conversely if $\int_{0}^{\infty}\left|H_{u}^{n}\right|^{2}$ du tends to zero in probability, then $\left(H^{n} \cdot \eta\right)^{*}$ tends to zero in probability.

Proof This follows from standard BDG inequalities and stopping time techniques, see [4] for details. The first step is to observe that $\int_{0}^{\infty}\left(H^{n}\right)^{\prime} \Sigma H^{n} d u$ tends to zero in probability. Then we use the previous lemma, i.e. assumption Delta, to conclude that this is equivalent to $\int_{0}^{\infty}\left(H^{n, j}\right)^{2} d u$ tending to zero in probability for each $j$. From here it follows that $\left(H^{n, j} \cdot \eta^{j}\right)_{\infty}$ tends to zero in probability. The converse follows from the fact that when $\langle H \cdot \eta, H \cdot \eta\rangle_{\infty}$ tends to zero in probability, stopping time techniques and the BDG inequalities imply that $(H \cdot \eta)^{*}$ tends to zero in probability.

Lemma 3 Under assumption Delta there is a martingale $\kappa=\left(\kappa^{1}, \ldots, \kappa^{d}\right)$ such that $d\left\langle\kappa^{i}, \eta^{k}\right\rangle=0$ for $i \neq k$ and $=d t$ for $i=k$. The process $\kappa$ also satisfies assumption Delta.

Proof The process $\kappa$ defined as $d \kappa=\Sigma^{-1 / 2} d B$ will do. The process is well defined and $d\langle\kappa, \eta\rangle=\Sigma^{-1 / 2} \Sigma^{1 / 2} d t$ as desired. The correlation matrix satisfies $d\left\langle\kappa, \kappa^{\prime}\right\rangle_{t}=(\Sigma)_{t}^{-1} d t$. This implies that the correlation matrix of $\kappa$ is uniformly bounded and is also uniformly elliptic.

\section{The Main Result, Applications to Risk Mea- surement}

We will now state and prove the main theorem.

Theorem 4 With the notations introduced in the first section we have that

$$
\sum_{j} W_{j}+L_{+}^{\infty}=\sum_{j} \mathcal{A}_{j}
$$

is norm dense in the set $\left\{f \in L^{\infty} \mid \mathbb{E}[f] \geq 0\right\}$.

Proof It is sufficient to prove that if $a \in L^{\infty}$ with $\mathbb{E}[a]=0,\|a\|_{\infty} \leq 1$, then $a$ is in the norm closure of $\sum_{j} \mathcal{A}_{j}$. This is the same as saying that for every $1 \geq \varepsilon>0$ we have that $a+\varepsilon \in \sum_{j} \mathcal{A}_{j}$. The proof will be done via a separation argument. Since the set $\sum_{j} \mathcal{A}_{j}$ is not norm closed we have to use a different space. The duality we will use is the duality between $\left(L^{1}\right)^{d}$ and $\left(L^{\infty}\right)^{d}$. Both spaces are equipped with the product topology. These spaces are in duality via the coupling $\phi(f)=\langle\phi, f\rangle=\sum_{j} \mathbb{E}\left[\phi_{j} f_{j}\right]$. We introduce the set

$$
\mathcal{D}_{n}=\left\{\left(f_{1}, \ldots, f_{d}\right) \mid\left\|f_{j}\right\|_{\infty} \leq n+2, \sum_{j} f_{j}=a+\varepsilon\right\} .
$$


The set $\mathcal{D}_{n}$ is convex and weak ${ }^{*}$ compact. Suppose that it is disjoint from the closed convex set $\prod_{j} \mathcal{A}_{j}$. Then we can find elements $\phi^{n} \in\left(L^{1}\right)^{d}$ such that

$$
\sup \left\{\sum_{j} \mathbb{E}\left[\phi_{j}^{n} f_{j}\right] \mid f \in \mathcal{D}_{n}\right\}<\inf \left\{\sum_{j} \mathbb{E}\left[\phi_{j}^{n} g_{j}\right] \mid g_{j} \in \mathcal{A}_{j}\right\} .
$$

Since the sets used on the right are cones, the infimum must be zero. This implies that the elements $\phi_{j}^{n}$ are nonnegative. Since the inequality is strict, one of the elements, say $\phi_{1}^{n}$, must be different from the zero element. So we can normalise this element and suppose that it is a probability density. Since for each $i \neq 1$ the element $f$ defined as $f_{j}=0$ for $j \neq i, 1, f_{1}=a+\varepsilon-h, f_{i}=h$ where $\|h\| \infty \leq n$ is in $\mathcal{D}_{n}$, we get that

$$
\mathbb{E}\left[\phi_{1}^{n}(a+\varepsilon)\right]+\sup \left\{\left(\phi_{1}^{n}-\phi_{i}^{n}\right) h \mid\|h\|_{\infty} \leq n\right\}<0 .
$$

Of course this yields $\mathbb{E}\left[\phi_{1}^{n}(a+\varepsilon)\right]<0$. Since we normalised $\phi_{1}^{n}$ this also implies that $\left\|\phi_{1}^{n}-\phi_{i}^{n}\right\|_{1} \leq(1+\varepsilon) / n \leq 2 / n$. This yields that $\left\|\phi_{i}^{n}\right\| \leq 3$ for all $n, i$. We now use that $\phi_{i}^{n}$ annihilates the space $W_{i}$. If we denote by $\kappa$ the process introduced in lemma 3 of section 2 , we can write $\phi_{i}^{n}=\mathbb{E}\left[\phi_{i}^{n}\right]+H^{n, i} \cdot \kappa$ where $H_{i}^{n, i}=0$ and where $H^{n, i} \cdot \kappa$ defines a uniformly integrable martingale. Now we will show that all $\phi^{j}$ tend to 1 in $L^{1}$. Fix $i \neq 1$. Since $\phi_{1}^{n}-\phi_{i}^{n}$ tends to zero, we must have that the bracket of $\left(H^{n, 1}-H^{n, i}\right) \cdot \kappa$ tends to zero. By lemma 2 and by the property Delta for $\kappa$, this implies that for each $i \neq 1$ :

$$
\int_{0}^{\infty}\left(H_{i}^{n, 1}\right)_{u}^{2} d u
$$

tends to zero. Since $H_{1}^{n, 1}=0$ it follows that $\int_{0}^{\infty}\left|H^{n, 1}\right|^{2} d u$ tends to zero. This in turn implies that $\phi_{1}^{n}$ tends to 1 in probability. Scheffé's lemma then implies that the convergence takes place in $L^{1}$. But then we get $\mathbb{E}[a+\varepsilon]=\lim _{n} \mathbb{E}\left[\phi_{1}^{n}(a+\varepsilon)\right] \leq$ 0 , a contradiction to $\mathbb{E}[a]=0$.

The contradiction shows that $\mathcal{D}_{n}$ cannot be disjoint from $\prod_{j} \mathcal{A}_{j}$ for $n$ big enough. As a consequence we get the existence of elements $g_{j} \in \mathcal{A}_{j}$ so that $\sum_{j} g_{j}=a+\varepsilon$.

Remark 5 We can ask whether it is possible to give bounds for the $L^{\infty}$ norm of the elements $g_{j}$. To do this we need quantitative estimates on the speed of convergence of the elements $\phi^{n}$. Such inequalities exist and were the topic of an earlier version of this paper.

Remark 6 For each $j$ we define a coherent utility function, see [1], via the formula $m_{j}(f)=\sup \left\{a \mid f-a \in \mathcal{A}_{j}\right\}$. The infimal convolution of $m_{j}, j=$ $1, \ldots, d$ is defined as

$$
\mu(f)=\left(m_{1} \square m_{2} \square \ldots \square m_{d}\right)(f)=\sup \left\{\sum_{j} m_{j}\left(g_{j}\right) \mid \sum_{j} g_{j}=f\right\} .
$$

The functionals $\mu, m_{j}$ are defined on $L^{\infty}$. From standard duality results it follows that the cone that defines $\mu$ is given by the norm closure of $\sum_{j} \mathcal{A}_{j}$. So we have proved the following result: 
Theorem 7 If the process $\eta$ satisfies assumption Delta, then $\mu(f)=\mathbb{E}[f]$.

We will elaborate on this at the end of the paper, where we will analyse the case $d=2$.

\section{Another Interpretation of the Infimal Convo- lution}

In this section we will give another interpretation of the infimal convolution. We will use two coherent utility functions. One is defined through a convex closed set of probability measures $\mathcal{P}$ and is denoted by $u$, i.e. $u(f)=\inf _{\mathbb{Q} \in \mathcal{P}} \mathbb{E}_{\mathbb{Q}}[f]$. The other one, denoted by $m$, is defined by the set $\mathbb{M}^{a}$ of absolutely continuous risk neutral measures of a continuous d-dimensional price process $S$. The economic agent is confronted with the following situation. He has a future wealth described by the bounded random variable $f$. The associated utility is then $u(f)$. Since he is able to make financial transactions he can improve his utility by adding to $f$ a random variable that is attainable at zero cost. If, conformal to previous sections, $W$ denotes

$$
W=\left\{(H \cdot S)_{\infty} \mid H \cdot S \text { bounded }\right\},
$$

the economic agent is interested in the quantity

$$
\tilde{u}(f)=\sup \{u(f+g) \mid g \in W\} .
$$

A little algebra allows us to change this expression into the infimal convolution of $u$ and $m$. Indeed, for every $g \in L^{\infty}$ we have, see [3]: $g-m(g)=z_{g}+h_{g}$ where $z_{g} \in W$ and $h_{g} \geq 0$, so we can write

$$
\begin{aligned}
(u \square m)(f) & =\sup \left\{u(f-g)+m(g) \mid g \in L^{\infty}\right\} \\
& =\sup \left\{u(f-g+m(g)) \mid g \in L^{\infty}\right\} \\
& =\sup \left\{u\left(f-z_{g}-h_{g}\right) \mid g \in L^{\infty}\right\} \\
& =\sup \{u(f-z) \mid z \in W\} \\
& =\tilde{u}(f) .
\end{aligned}
$$

It follows that as soon as $\mathcal{P}$ is weakly compact, $\tilde{u}$ has the Fatou property, which in our setting means $\left\{f \in L^{\infty} \mid \tilde{u}(f) \geq 0\right\}$ is weak* closed, see [1] for more details. As we will see, without the weak compactness assumption, the property no longer holds.

\section{The Counterexample}

We now give a counterexample for the general case, i.e. the case where the correlation between two driving forces can approach 1 . The counterexample has its own interest since it is related to correlation trading. To make the calculations 
easier we will use two processes $S^{1}$ and $S^{2}$ to build the spaces $W_{1}, W_{2}$. The time interval is restricted to $[0,1]$. The movement of $S=\left(S^{1}, S^{2}\right)$ is given by

$$
\begin{aligned}
d S_{t}^{1} & =d B_{t}^{1} \\
d S_{t}^{2} & =d B_{t}^{1}+\epsilon_{t} d B_{t}^{2} .
\end{aligned}
$$

here $\epsilon$ is a deterministic function, rapidly decreasing to zero as $t \rightarrow 1$. We can take $\epsilon_{t}=\exp \left(-\frac{1}{1-t}\right)$. We do not normalise the movement of $S^{2}$ to have $d\left\langle S_{t}^{2}, S_{t}^{2}\right\rangle=d t$, This would only complicate the write-up and it is not essential. We denote by $\mathbb{M}_{1}^{a}$ and $\mathbb{M}_{2}^{a}$ the absolutely continuous probability measures that turn resp. $S^{1}$ and $S^{2}$ into a local martingale. The sets $\mathbb{M}_{1}^{a}$ and $\mathbb{M}_{2}^{a}$ can be decribed as the closures of:

$$
\begin{aligned}
& \mathbb{M}_{1}^{e}=\left\{\mathcal{E}\left(H \cdot B^{2}\right)_{1} \mid \int_{0}^{1} H_{u}^{2} d u<+\infty \text { a.s., } \mathbb{E}\left[\mathcal{E}\left(H \cdot B^{2}\right)_{1}\right]=1\right\} \\
& \mathbb{M}_{2}^{e}=\left\{\mathcal{E}\left(\left(H^{1}, H^{2}\right) \cdot\left(B^{1}, B^{2}\right)\right)_{1} \mid \int_{0}^{1} H_{u}^{2} d u<+\infty \text { a.s. },\right. \\
&\left.\mathbb{E}\left[\mathcal{E}\left(\left(H^{1}, H^{2}\right) \cdot\left(B^{1}, B^{2}\right)\right)_{1}\right]=1 \text { and } H_{t}^{1}+\epsilon_{t} H_{t}^{2}=0\right\} .
\end{aligned}
$$

The closures of these sets in the dual of $L^{\infty}$ are denoted by resp. $\mathcal{P}_{1}^{\text {ba }}$ and $\mathcal{P}_{2}^{\text {ba }}$. We easily see that $\mathbb{M}_{1}^{a} \cap \mathbb{M}_{2}^{a}=\{\mathbb{P}\}$ but we will show that

$$
\mathcal{P}_{1}^{\text {ba }} \cap \mathcal{P}_{2}^{\text {ba }} \neq\{\mathbb{P}\} .
$$

This means that the norm-closure of the set $\mathcal{A}_{1}+\mathcal{A}_{2}$ is strictly smaller than the set $\left\{a \in L^{\infty} \mid \mathbb{E}[a] \geq 0\right\}$. Let us take the following sequence of stochastic exponentials:

$$
L^{n}=\mathcal{E}\left(H^{n} \cdot B^{2}\right),
$$

where $H^{n}=-(5 / 2)^{n} \mathbf{1}_{\left.11-2^{-n}, 1-2^{-(n+1)}\right]}$. This sequence is in $\mathbb{M}_{1}^{e}$. The sequence $\left(L_{1}^{n}\right)_{n \geq 1}$ is equivalent to the standard basis in $l^{1}$. This means that there is a $\delta>0$ so that $\left\|\sum_{n} \alpha_{n} L_{1}^{n}\right\|_{1} \geq \delta \sum_{n}\left|\alpha_{n}\right|$ for all sequences $\left(\alpha_{n}\right)_{n}$ with only finitely many $\alpha_{n} \neq 0$. (As the reader can verify the sequence $L_{1}^{n}$ is supported by "almost" disjoint sets). Therefore its adherent points all lie in (ba $\left.\backslash L^{1}\right)$ (see below). The set of these adherent points is quite big. In fact it is homeomorphic to the compact set $\beta(\mathbb{N}) \backslash \mathbb{N}$, where $\beta(\mathbb{N})$ is the Stone-Čech compactification of $\mathbb{N}$. Now look at the sequence $\mathcal{E}\left(\left(-\epsilon H^{n}, H^{n}\right) \cdot\left(B^{1}, B^{2}\right)\right)_{1}$. This is a sequence in $\mathbb{M}_{2}^{a}$. This sequence has the same weak* adherent points (in ba) as the sequence $\mathcal{E}\left(H^{n} \cdot B^{2}\right)_{1}$. This is proved by calculating the $L^{1}$-norm of their difference

$$
\left\|\mathcal{E}\left(H^{n} \cdot B^{2}\right)_{1}-\mathcal{E}\left(\left(-\epsilon H^{n}, H^{n}\right) \cdot\left(B^{1}, B^{2}\right)\right)_{1}\right\|_{1} .
$$

If we denote by $\mathbb{Q}^{n}$ the measure (by the way in $\mathbb{M}_{1}^{e}$ ) defined as $d \mathbb{Q}^{n} / d \mathbb{P}=$ $\mathcal{E}\left(H^{n} \cdot B^{2}\right)_{1}$, the above expression is simply

$$
\mathbb{E}_{\mathbb{Q}^{n}}\left[\left|\mathcal{E}\left(-\epsilon H^{n} \cdot B^{1}\right)_{1}-1\right|\right]
$$


Since under $\mathbb{Q}^{n}$ the process $B^{1}$ is still a Brownian motion and since all the integrands are deterministic, we get that the expression is the same as

$$
\mathbb{E}_{\mathbb{P}}\left[\left|\mathcal{E}\left(-\epsilon H^{n} \cdot B^{1}\right)_{1}-1\right|\right]
$$

Since $\epsilon H^{n}$ tends to zero uniformly on $[0,1] \times \Omega$, we get that the above expression tends to zero. So the adherent points in ba of the sequences $\mathcal{E}\left(H^{n} \cdot B^{2}\right)_{1}$ and $\mathcal{E}\left(\left(-\epsilon H^{n}, H^{n}\right) \cdot\left(B^{1}, B^{2}\right)\right)_{1}$ are the same and consequently the sets $\mathcal{P}_{1}^{\text {ba }}$ and $\mathcal{P}_{2}^{\text {ba }}$ have an intersection that is much bigger than $\{\mathbb{P}\}$.

Lemma 8 Let $E$ be a Banach space and let $F \subset E$ be a closed subspace of $E$, isomorphic to the space $l^{1}$. Let $\left(y_{n}\right)_{n \geq 1}$ be the sequence in $F$ that corresponds to the unit vector base of $l^{1}$. Let $e^{* *} \in E^{* *}$ be a weakly adherent point of the sequence $\left(y_{n}\right)_{n \geq 1}$. Then $e^{* *} \in E^{* *} \backslash E$.

Proof. Let $T$ be an isomorphism between $F$ and $l^{1}$, so that $T\left(y_{n}\right)$ is the unit vector base of $l^{1}$. In case $e^{* *} \in E$, we would have that for every $k$ the point $e^{* *}$ would be in the weak closure, i.e. $\sigma\left(E, E^{*}\right)$, of the convex hull of the sequence $\left(y_{n}\right)_{n \geq k}$. By the Hahn-Banach theorem it is therefore in the norm closure of these convex hulls. This would mean that there are convex combinations $z_{k}$ of $\left(y_{n}\right)_{n \geq k}$ that would converge in norm to $e^{* *}$. It also means that $e^{* *} \in F$. Translated by $T$ this means that the basic sequence of $l^{1}$ has an adherent point in $l^{1}$, an obvious contradiction. Therefore $e^{* *} \in E^{* *} \backslash E$.

\section{Further analysis of the case $d=2$}

We start with a two dimensional standard Brownian Motion $B=\left(B^{1}, B^{2}\right)$. Let us define the sets of probability measures (denoted by $\mathbb{M}_{1}^{a}$, resp. $\mathbb{M}_{2}^{a}$ ) so that $B^{1}$, resp. $B^{2}$, is still a Brownian Motion. In mathematical finance this means that these sets are the risk neutral probability measures for the "stock prices", $B^{1}$, resp. $B^{2}$. The coherent utility functions defined with these sets are denoted by resp. $m_{1}$ and $m_{2}$, see [1] for the details. This means that for a function $f \in L^{\infty}$ we have $m_{1}(f)=\inf \left\{\mathbb{E}_{\mathbb{Q}}[f] \mid \mathbb{Q} \in \mathbb{M}_{1}^{a}\right\}$ and $m_{2}(f)=\inf \left\{\mathbb{E}_{\mathbb{Q}}[f] \mid \mathbb{Q} \in \mathbb{M}_{2}^{a}\right\}$. Both have the Fatou property, see [1] for details. The sets $\mathbb{M}_{1}^{a}$ and $\mathbb{M}_{2}^{a}$ can be decribed as the $L^{1}$-closures of:

$$
\begin{aligned}
& \mathbb{M}_{1}^{e}=\left\{\mathcal{E}\left(H \cdot B^{2}\right)_{\infty} \mid \int_{0}^{\infty} H_{u}^{2} d u<+\infty \text { a.s., } \mathbb{E}\left[\mathcal{E}\left(H \cdot B^{2}\right)_{\infty}\right]=1\right\} \\
& \mathbb{M}_{2}^{e}=\left\{\mathcal{E}\left(H \cdot B^{1}\right)_{\infty} \mid \int_{0}^{\infty} H_{u}^{2} d u<+\infty \text { a.s., } \mathbb{E}\left[\mathcal{E}\left(H \cdot B^{1}\right)_{\infty}\right]=1\right\}
\end{aligned}
$$

where of course the integrands $H$ are predictable. These sets are not relatively weakly compact. The closures of these sets in the dual ba of $L^{\infty}$ are denoted by resp. $\mathcal{P}_{1}^{\text {ba }}$ and $\mathcal{P}_{2}^{\text {ba }}$. The question that now arises is whether the infimal convolution, $m_{1} \square m_{2}$, has the Fatou property. As in [1] one can easily see that $m_{1} \square m_{2}$ has the Fatou property if and only we have $\mathcal{P}_{1}^{\text {ba }} \cap \mathcal{P}_{2}^{\text {ba }}=\{\mathbb{P}\}$. This 
follows from standard duality arguments which we do not repeat. The equality to be used is $m_{1} \square m_{2}(f)=\inf \left\{\mu(f) \mid \mu \in \mathcal{P}_{1}^{\text {ba }} \cap \mathcal{P}_{2}^{\text {ba }}\right\}$. That

$$
\mathbb{M}_{1}^{a} \cap \mathbb{M}_{2}^{a}=\{\mathbb{P}\}
$$

is fairly obvious. But what happens with $\mathcal{P}_{1}^{\text {ba }} \cap \mathcal{P}_{2}^{\text {ba }}$ ? The problem is equivalent to the approximation property of section 2 as we shall now explain. Let us recall

$$
\begin{aligned}
& W_{1}=\left\{\left(H \cdot B^{1}\right)_{\infty} \mid H \text { predictable and }\left(H \cdot B^{1}\right) \text { bounded }\right\} \\
& W_{2}=\left\{\left(H \cdot B^{2}\right)_{\infty} \mid H \text { predictable and }\left(H \cdot B^{2}\right) \text { bounded }\right\}
\end{aligned}
$$

Furthermore let us also recall, see [3], [2], that:

$$
\begin{aligned}
& \mathcal{A}_{1}=\left\{f+g \mid f \in W_{1} \text { and } 0 \leq g \in L^{\infty}\right\}=\left\{h \mid \mathbb{E}_{\mathbb{Q}}[h] \geq 0 \text { for all } \mathbb{Q} \in \mathbb{M}_{1}^{e}\right\} \\
& \mathcal{A}_{2}=\left\{f+g \mid f \in W_{2} \text { and } 0 \leq g \in L^{\infty}\right\}=\left\{h \mid \mathbb{E}_{\mathbb{Q}}[h] \geq 0 \text { for all } \mathbb{Q} \in \mathbb{M}_{2}^{e}\right\} .
\end{aligned}
$$

We will prove, or simply remark, that

1. $\mathcal{A}_{1}=\left\{f \mid m_{1}(f) \geq 0\right\}$ and $\mathcal{A}_{2}=\left\{f \mid m_{2}(f) \geq 0\right\}$.

2. The norm closure of $\mathcal{A}_{1}+\mathcal{A}_{2}$ (or of $W_{1}+W_{2}+L_{+}^{\infty}$ ) equals the set $\{f \mid$ $\left.m_{1} \square m_{2}(f) \geq 0\right\}$. This is a standard duality result and follows from the Hahn-Banach theorem.

3. The set $W_{1}+W_{2}$ is not norm-closed. The cone $\mathcal{A}_{1}+\mathcal{A}_{2}$ is not norm closed.

4. The norm closure of $W_{1}+W_{2}$ is strictly contained in the set

$$
L_{0}^{\infty}=\left\{f \in L^{\infty} \mid \mathbb{E}_{\mathbb{P}}[f]=0\right\} .
$$

Of course the representation theorem for martingales shows that the set $W_{1}+W_{2}$ is weak* dense in $L_{0}^{\infty}$.

5. $\mathcal{P}_{1}^{\text {ba }} \cap \mathcal{P}_{2}^{\text {ba }}=\{\mathbb{P}\}$. This means that the set $\mathcal{A}_{1}+\mathcal{A}_{2}$ is norm-dense in $\{f \mid \mathbb{E}[f] \geq 0\}$. This is precisely the content of theorems 4 and 7. Another way to state the same result is to say that the norm closure of the set $\mathcal{A}_{1}+\mathcal{A}_{2}$ is weak ${ }^{*}$ closed. There is a big difference when compared to the norm closure of the set $W_{1}+W_{2}$.

6. $m_{1} \square m_{2}=\mathbb{P}$. This is equivalent to $\mathcal{P}_{1}^{\text {ba }} \cap \mathcal{P}_{2}^{\text {ba }}=\{\mathbb{P}\}$.

7. By item 3 and 4 , there are non-trivial elements $\mu \in \mathbf{b a} \backslash L^{1}$ that are zero on $W_{1}+W_{2}$ but item 5 shows that there are no non-trivial nonnegative elements $\mu \in$ ba $\backslash L^{1}$ that are zero on $W_{1}+W_{2}$.

The proofs of 1, 2, 5, 6 and 7 are already sketched or are obvious. The proof of 3 is given below. To prove 4 we need some extra information on BMO martingales.

Proposition 9 Let $T=\inf \left\{t|| B_{t}^{1}+B_{t}^{2} \mid=1\right\}$. The stopping time $T$ has a Laplace transform given by $\mathbb{E}\left[\exp \left(-\lambda^{2} T\right)\right]=1 / \cosh (\lambda)$. For $s \in \mathbb{C},|\Re(s)|<\pi / 2$ we have $\mathbb{E}\left[\exp \left(s^{2} T\right)\right]=1 / \cos (s)$. Also $\mathbb{E}\left[\exp \left((\pi / 2)^{2} T\right)\right]=+\infty$. 
Proof That $B_{T}^{1}$ and $B_{T}^{2}$ are unbounded is easily seen, we leave the proof to the reader. The Laplace transform of $T$ is found by standard methods. Let us look at the martingale $M_{t}=\exp \left(\lambda\left(B_{t}^{1}+B_{t}^{2}\right)-\lambda^{2} t\right)$. Then by symmetry the variable $a=B_{T}^{1}+B_{T}^{2}$ has a distribution given by $\mathbb{P}[a=1]=\mathbb{P}[a=-1]=1 / 2$. Moreover by symmetry, the variable $a$ is independent of $T$. Since for $t \leq T, M_{t} \leq \exp (|\lambda|)$ we can apply the stopping time theorem and we get $\mathbb{E}\left[\exp \left(\lambda a-\lambda^{2} T\right)\right]=1$. This immediately implies $\mathbb{E}\left[\exp \left(-\lambda^{2} T\right)\right]=1 / \cosh (\lambda)$. Since $\cosh (z)$ is analytic around the origin and different from 0 for $|z|<\pi / 2$ we get for $z$ complex and for $|\Re(z)|<\pi / 2$ that $\mathbb{E}\left[\exp \left(z^{2} T\right)\right]=1 / \cosh (i z)=1 / \cos (z)$. In particular this holds for $s \in \mathbb{R},|s|<\pi / 2$. Moreover we get that $\mathbb{E}\left[\exp \left((\pi / 2)^{2} T\right)\right]=+\infty$ as an application of the monotone convergence theorem for $s \rightarrow \pi / 2$.

Proposition 10 The variable $B_{T}^{1}$ satisfies $\mathbb{E}\left[\exp \left(\alpha B_{T}^{1}\right)\right]=+\infty$ for $\alpha \geq \pi$. The random variable $B_{T}^{1}$ is in $B M O$ but not in the closure of $L^{\infty}$ in $B M O$.

Proof The two processes $B^{1}+B^{2}$ and $B^{1}-B^{2}$ are independent processes. Furthermore the stopping time $T$ is defined through $B^{1}+B^{2}$ and hence independent of $B^{1}-B^{2}$. Let us denote by $\mathcal{G}$ the $\sigma$-algebra generated by the process $B^{1}+B^{2}$. We then get:

$$
\mathbb{E}\left[\exp \left(2 \lambda B_{T}^{1}\right)\right]=\mathbb{E}\left[\mathbb{E}\left[\exp \left(\lambda\left(B^{1}-B^{2}\right)_{T}\right) \mid \mathcal{G}\right] \exp \left(\lambda\left(B^{1}+B^{2}\right)_{T}\right)\right]
$$

which equals

$$
\mathbb{E}\left[\exp \left(\lambda^{2} T\right) \exp (\lambda a)\right] .
$$

Since $a$ and $T$ are independent this gives

$$
\cosh (\lambda) \mathbb{E}\left[\exp \left(\lambda^{2} T\right)\right] .
$$

For $\lambda \geq \pi / 2$ this is $+\infty$. The statement about $B M O$ follows from $B M O$-theory where it is shown - by the John-Nirenberg inequality - that elements in the closure of $L^{\infty}$ in $B M O$ necessarily have exponential moments of all order. We do not give details since this is beyond the scope of this paper.

Corollary 11 The above calculations together with proposition 9, also show that for $|\lambda|<\pi / 2$ we have $\mathbb{E}\left[\exp \left(2 \lambda B_{T}^{1}\right)\right]=\cosh (\lambda) / \cos (\lambda)$. The characteristic function of $2 B_{T}^{1}$ is therefore $\mathbb{E}\left[\exp \left(i \lambda 2 B_{T}^{1}\right)\right]=\cos (\lambda) / \cosh (\lambda)$ and the characteristic function of $B_{T}^{1}-B_{T}^{2}$ equals $1 / \cosh (\lambda)$.

Proposition 12 The variable a defined above cannot be in the BMO-closure of $W_{1}+W_{2}$, hence the variable a cannot be in the $L^{\infty}$-closure of $W_{1}+W_{2}$.

Proof Suppose that $a$ would be in the closure of $W_{1}+W_{2}$ for the $B M O$ topology. This means that there are $f^{n} \in W_{1}$ and $g^{n} \in W_{2}$ so that $f^{n}+g^{n} \rightarrow a$. It can be shown that this implies that $f^{n} \rightarrow B_{T}^{1}$ in $B M O$. Since $B_{T}^{1}$ is not in the $B M O$ closure of $L^{\infty}$ this is a contradiction.

Remark 13 As shown in the main theorem the element $a$ is in the norm-closure of the set $\mathcal{A}_{1}+\mathcal{A}_{2}$. This means that for every $\epsilon>0$ there are elements $f \in$ $W_{1}, g \in W_{2}$ so that $f+g \leq \epsilon+a$. Similarly there are also elements $f^{\prime} \in W_{1}, g^{\prime} \in$ $W_{2}$ so that $f^{\prime}+g^{\prime} \geq-\epsilon+a$. 
We can now complete the proof of items 3 and 4 in the beginning of this section.

Proposition 14 The set $W_{1}+W_{2}$ is not norm closed.

Proof Since $W_{1} \cap W_{2}=\{0\}$ the closedness of $W_{1}+W_{2}$ would imply - by the closed graph theorem - that the projections $W_{1}+W_{2} \rightarrow W_{i}$ would be continuous. Let us define $T_{n}=\inf \left\{t|| B_{t}^{1} \mid \geq n\right\} \wedge T$ where $T$ is defined in the previous propositions. Set $a^{n}=\mathbb{E}\left[a \mid \mathcal{F}_{T_{n}}\right]=B_{T_{n}}^{1}+B_{T_{n}}^{2}$. Then clearly $\left\|a^{n}\right\|_{\infty} \leq 1$ but as easily seen $\left\|B_{T_{n}}^{1}\right\|_{\infty}=n$. This implies that the projections $W_{1}+W_{2} \rightarrow W_{i}$ cannot be continuous. Therefore $W_{1}+W_{2}$ is not closed.

Remark 15 This also means that there are sequences $f_{n} \in W_{1}$ and $g_{n} \in W_{2}$ so that $\left\|f_{n}\right\|_{\infty}=\left\|g_{n}\right\|_{\infty}=1$ and such that $\left\|f_{n}-g_{n}\right\|_{\infty} \rightarrow 0$. For instance we can take $f_{n}=\frac{B_{T_{n}}^{1}}{n}$ and $g_{n}=\frac{-B_{T_{n}}^{2}}{n}$.

Proposition 16 The set $\mathcal{A}_{1}+\mathcal{A}_{2}$ is not norm closed.

Proof If the set $\mathcal{A}_{1}+\mathcal{A}_{2}$ were norm closed then the set $W_{1}+W_{2}=\{b \mid b \in$ $\mathcal{A}_{1}+\mathcal{A}_{2}$ and $\left.\mathbb{E}[b]=0\right\}$ would also be norm closed.

Proposition 17 The following equation holds for every $f \in L^{\infty}$.

$$
\mathbb{E}[f]=\sup _{g \in W_{2}} \inf _{\mathbb{Q} \in \mathbb{M}_{1}^{a}} \mathbb{E}_{\mathbb{Q}}[f+g]=\sup _{g \in W_{1}} \inf _{\mathbb{Q} \in \mathbb{M}_{2}^{a}} \mathbb{E}_{\mathbb{Q}}[f+g] .
$$

Proof This follows from $m_{1} \square m_{2}=\mathbb{E}[$.$] and the interpretation of the infimal$ convolution as described in section 4 .

Remark 18 Most of the above results can be generalised to the case where the two Brownian motions are correlated but satisfy assumption Delta. We leave this as an exercise.

\section{References}

[1] Delbaen, F. Coherent Risk Measures, Scuola Normale Superiore di Pisa, 2000

[2] Delbaen, F. Representing Martingale Measures when Asset Prices are Continuous and Bounded., Mathematical Finance 2, 1992, pp. 107-130.

[3] Delbaen, F. and Schachermayer, W. A general version of the fundamental theorem of asset pricing, Math. Ann. 300, 1994, pp. 463-520.

[4] D. Revuz and M. Yor: Continuous Martingales and Brownian Motion, Springer Verlag, Berlin Heidelberg New York, 1991 